\title{
PERSPECTIVE
}

\section{Digital devices and continuous telemetry: opportunities for aligning psychiatry and neuroscience}

\author{
Justin T. Baker ${ }^{1}$, Laura T. Germine ${ }^{1}$, Kerry J. Ressler ${ }^{1}$, Scott L. Rauch ${ }^{1}$ and William A. Carlezon Jr. (D) \\ Neuropsychopharmacology (2018) 43:2499-2503; https://doi.org/10.1038/s41386-018-0172-z
}

\begin{abstract}
OVERVIEW
Here we provide perspective on the past, present, and future of rapidly emerging technologies-digital devices and continuous telemetry-in the study and treatment of psychiatric illness from human patients to animal models. We focus on how commonly available technologies are helping to establish a robust data infrastructure in both clinical and research settings that has nearterm potential to improve the precision in our ability to measure, monitor, and model behavior across a range of clinical, naturalistic, and basic research settings. We also forecast how these approaches and technical capabilities are likely to converge to the benefit of humans with neuropsychiatric conditions over the next five years.

The word "technology" comes from the Greek "techne" for art or skill, and "logia", implying the systematic study or treatment, which together signifies the systematic study or application of an art or craft. Psychiatry derives from "psyche" or mind, but with a different Greek suffix ("iatria"), which means healing rather than study (as in "psychology" and "neurology"). If the essence of psychiatry is healing the mind, the goal of technology in psychiatry is to systematize the art of mind healing. From the mental status exam (MSE), the Diagnostic and Statistical Manual (DSM) and the International Classification of Disease (ICD) system, to manualized psychotherapies and treatment algorithms, the field has explored many approaches to systematizing the complex art of diagnosis, treatment, and prevention of neuropsychiatric conditions. Throughout this evolution, the tension between systems that work well at cohort and population scale by minimizing individual differences and those that work well for individuals has resulted in modest progress in care processes and outcomes. In this context, we focus on scaleable technologies intended to enhance the care experience and outcomes, providing platforms that can incorporate insights from prior traditions through a combination of measurement and systematic delivery of individualized therapy.
\end{abstract}

\section{PAST}

Ten years ago, smart phone usage and ownership among the general public, let alone patients with severe psychiatric illness, was limited. This gap in access-the so-called "digital divide"meant that even though technology was available to remotely monitor individuals with complex conditions, many solutions relied on additional devices to support remote monitoring solutions. For instance, in 2007, the Bosch company introduced a telehealth service (Health Buddy), which used an internet-connected device with a simple 4-button interface to allow for patient prompts for conditions including depression. Despite the simplicity of the interface, this technology failed to create sustainable engagement. Also around this time, the global mental health crisis was driving considerable interest in solutions that incorporated technology to reach young people suffering from mental illness in both the developed and developing world [1].

Five years ago, the proliferation of smart phones and wearable devices led several software companies to form businesses around the smart phone itself as the remote monitoring platform. These companies leveraged increasing smart phone ownership with the needs of providers and healthcare system administrators to create dashboards that could capture patient acuity across a clinic or an entire system, while also providing therapeutic content and secure communications. In parallel, several groups developed internetbased cognitive behavioral therapy applications that showed promise in treating depression and anxiety [2]. While such systems continue to improve and gain traction in some clinical settings, platform engagement for both patients and providers has limited the uptake of these services and caused some of these companies to pivot their business models. For instance, one such company (Ginger.io) has re-focused on delivering coaching through their software platform with a direct-to-consumer focus, rather than being a product geared for providers.

In parallel, electronic health record (EHR) systems have gained increasing traction over the past decade; according to a CDC survey, $>87 \%$ of physician practices use some form of EHR. The push for additional documentation and measurement afforded by EHR systems has both changed the landscape in terms of what devices and other software are needed to fill service gaps, but also in tempering enthusiasm among psychiatrists for whether technology in general is a help or hindrance to their practice. Psychiatry has the lowest EHR adoption of any specialty, with $\sim 60 \%$ of psychiatrists using an EHR [3], even while the EHR data has been shown to provide predictive utility in risk stratifying psychiatric patients, such as identifying individuals who are more likely to make a suicide attempt following an evaluation [4, 5]. While substantial skepticism remains about the utility of EHRs in psychiatry, the American Psychiatric Association now devotes a webpage to the topic, including information about EHRs geared toward mental health providers (https://www.capterra.com/ mental-health-software/). Moreover, we recently proposed several ways the federal government could improve adoption of EHRs in psychiatric care to meet the goals of the $21^{\text {st }}$ Century Cures Act [6].

Citations for papers with the terms "digital technology" and "psychiatry" are ramping up in ways that resemble trends for

${ }^{1}$ Department of Psychiatry, Harvard Medical School, McLean Hospital, Belmont, MA, USA

Correspondence: William A. Carlezon Jr. (bcarlezon@mclean.harvard.edu)

Received: 23 May 2018 Revised: 26 July 2018 Accepted: 27 July 2018

Published online: 2 August 2018 
"fMRI" and "psychiatry" at the turn of the century (Fig. 1). Signs suggest that digital approaches will take the same exponential trajectory, if not potentially even more expansive given these approaches are more likely to gain traction in clinical practice in ways that neuroimaging was never well suited to realize, in part due to the lack of portability of the devices.

\section{CURRENT}

In 2018, psychiatrists and researchers now have many options for incorporating technology and digital devices into the management and study of psychiatric illnesses that go beyond what is provided by the EHR. Our culture has acclimated to the pervasiveness of personal computing devices, with $>75 \%$ of US adults carrying smart phones and $>90 \%$ using the internet; globally, the number of smart phone users will soon surpass 2.5 billion, with $48 \%$ able to access the internet. With this evolution has come increasing expectations for how technology should help us stay organized, communicate, stay mentally fit, and track our physical health. While today's device landscape contains gadgets perched between being useful versus distracting, connecting vs. isolating, there is little doubt that in the right settings, and with appropriate guidance and support, technology can improve the quality of psychiatric care for a tremendous number of people in need [7].

The push to integrate mental and behavioral health care into primary care has led to numerous advances in systematizing treatments. This is particularly important for people with dementia or severe mental illness, who may lose key mental faculties at times they are not around caretakers, or refuse a conventional Mental Status Exam (MSE). New metrics and approaches derived from digital devices have emerged in the past few years that should augment the conventional toolkit for human behavioral evaluation in naturalistic settings. Some of these metrics derive from smart phones and wearable fitness trackers, and allow researchers to ascertain key parameters of psychiatric health, including sleep patterns and overall activity levels, but also more nuanced metrics of mental state that could potentially recapitulate every aspect of the traditional MSE (Fig. 2). Another promising set of metrics derive from quantitative analysis of behavior during in-person evaluations, using video and computer vision to automatically code facial expressions, gaze, pose and postural dynamics that can inform clinical assessment for a range of conditions, including depression [8, 9], psychotic disorders [10], and autism [11]. These approaches have the advantage of retaining existing (and trusted) workflows. They emphasize the importance of face-to-face communication-a preferable form of interaction for many individuals-while bringing systematic evaluation through computer vision of behavior and acoustic analysis of speech in more conventional clinical settings.

Continuous data sets of these metrics may enable a better understanding of what people are doing before the signs and symptoms become evident-perhaps even before the patients themselves are aware-and thus help to avoid crises. For instance, application of continuous digital metrics have already been shown to have predictive value for estimating which individuals will develop worsening pathology, including conversion to psychosis $[12,13]$, re-attempt of suicide after a presentation to the emergency room with an initial attempt [14], or relapse during schizophrenia treatment requiring hospitalization [15]. These examples are among the first to show utility in what is likely to be a rapid expansion of predictive digital phenotyping. While exciting, the ability to anticipate and therefore prevent adverse outcomes is fraught with ethical challenges: questions include when and for whom it is appropriate to take preventative actions on the basis of these signals, and to what extent individual autonomy would be threatened or potentially preserved by increasingly accurate prevention technologies covering a range of mental health conditions and outcomes. By benchmarking against real-world events, including those selected by the individual seeking care, such approaches also have the potential to establish entirely new metrics with predictive value that do not necessarily map onto conventional assessments, but may be better suited to individual needs and definitions of what consistitutes treatment success.

\section{FUTURE}

Much as occurred after the initial phase of neuroimaging, we anticipate that research groups without strong technical expertise to adopt these approaches to explore the full range of clinical prediction models in robust data-rich studies, such as those aimed to prevent suicide or relapse to drug seeking in at-risk individuals. We also expect some new technologies to surpass what is

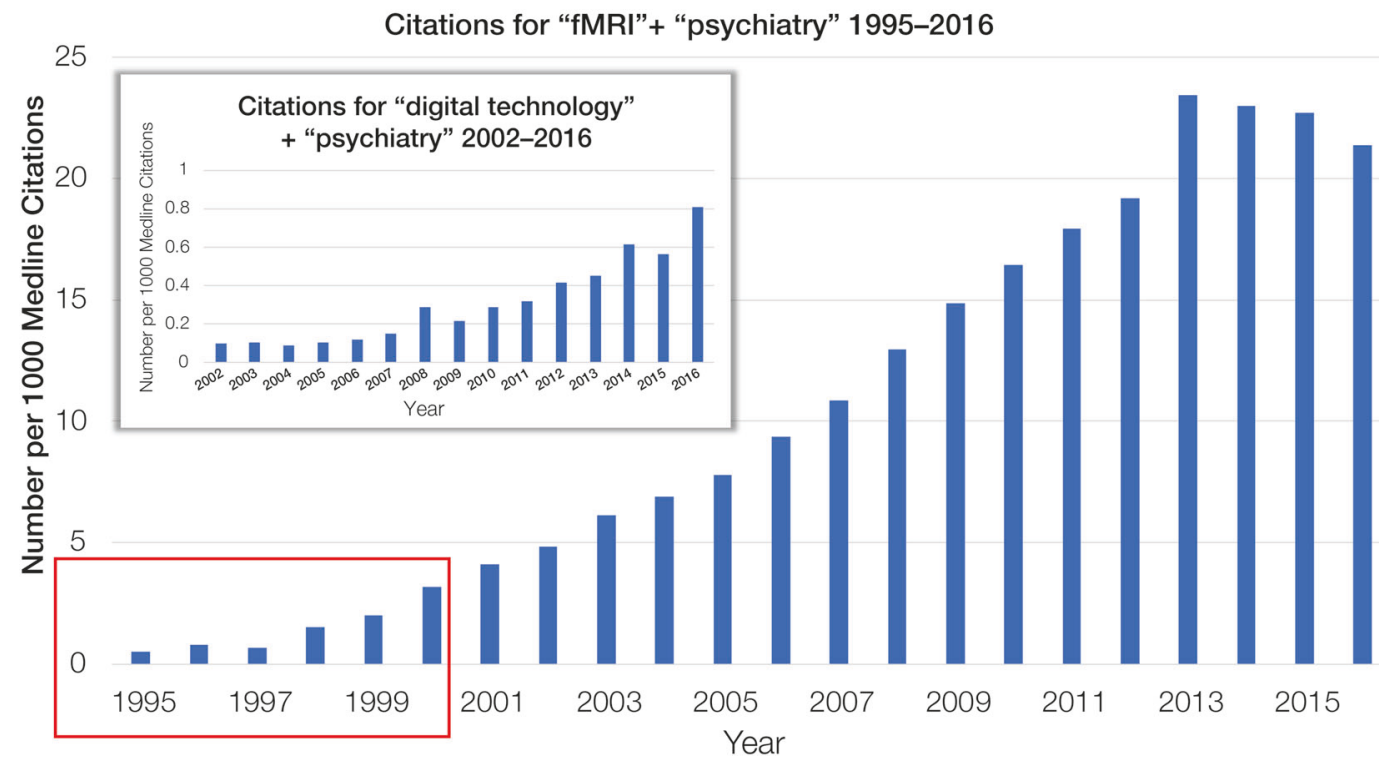

Fig. 1 Citations for the terms "digital technology" and "psychiatry" over the past five years show similar trends as the terms "fMRI" and "psychiatry" in 1999, expressed as number per 1000 Medline citations 


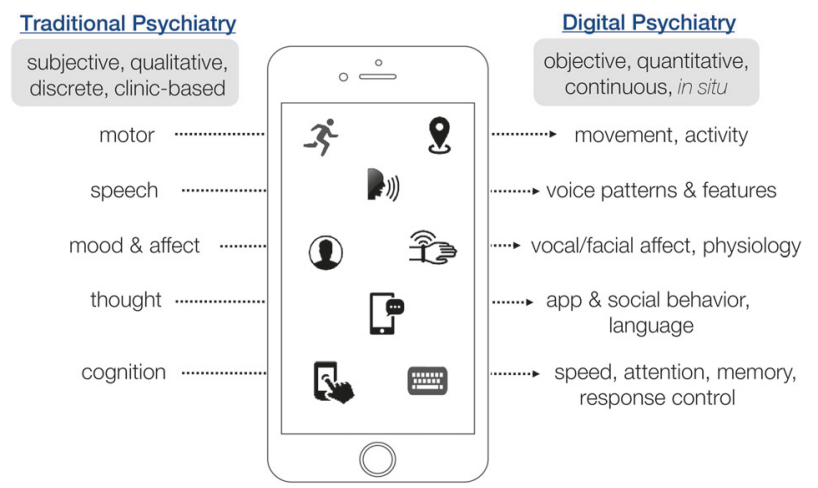

Fig. 2 Revisiting the Mental Status Exam (MSE) in an era of digital phenotyping. Traditional psychiatry may undergo a radical transformation of the most fundamental means of assessment, as digital tools pave the way for more efficient, naturalistic capture of an individual's mental status through continuous analysis of devicebased interactions. Figure created by S. Rauch, L. Germine \& N. Mirin, and presented at the Technology in Psychiatry Summit, Boston MA, USA, 06 Nov 2017; https://www.youtube.com/watch? $\mathrm{v}=\mathrm{gFwN08HzRwMt}=42 \mathrm{~s}$

currently available to clinicians and researchers as mainstays of the clinical and research pipelines. The next five years are likely to bring an increasingly sensor-rich environment to human societyin cars, homes, and everyday objects-replete with cameras, microphones, and sensors to measure our interactions, activities, and physiology in situ. For a system that measures continuous, temporally dense behavior in an individual's natural setting to be widely adopted, it must be capable of providing useful, unobtrusive behavioral feedback and a controlled environment that meets and anticipates individualized needs. Such systems are emerging, with the FDA recently approving the first smart watch for remote monitoring of a neuropsychiatric condition and the first app to be marketed for treatment of substance use disorders.

Under development, wearable devices that can detect or stimulate brain and body, such as ambulatory EEG or tDCS, are likely to provide the ability to create closed-loop systems for influencing brain circuits in real-time, in response to contextual signals derived from individual behavior, brain, or environmental signals. Autonomous dosing systems, similar to the insulin pump but for psychiatric drugs and neurostimulatory therapies, will become increasingly feasible, as the signals for measuring whether they have helped or hurt are increasingly captured by remote sensor networks.

Finally, computational approaches to analyze these kind of individualized data already exist, are used heavily in other fields, and will translate well to psychiatry once suitable data sets are available. Companies like Netflix or Apple thrive on their ability to provide services and recommendations based on crowd-sourced insights, derived from large user data bases and longitudinal behavioral data about preferences aggregated from each user's interactions with their platform as relate to other aspects of their profile. These algorithms derive from non-parametric Bayesian statistics, which can account for missingness and variable usage patterns, which are inherent to any longitudinal, multivariate data collected from humans in naturalistic settings. As the rich clinical and behavioral data emerge from the many technologies described above make their way into daily life and common clinical practice, these same approaches will yield progressively less missingness and increased complexity. Fortunately, when combined with content expertise and human intuition, computational approaches can disentangle this richness and complexity, driving insights about the structure and dynamics of human behavior while enabling radical transformations in how we detect, respond to, and anticipate psychopathological states. What is needed to achieve this goal is sufficient data from real individuals to inform and improve modeling.

\section{COMPLEMENTARY STUDIES IN LABORATORY ANIMALS}

The rapid evolution of technology has also affected another domain that influences mental health: basic neuroscience research. There has been a parallel evolution of molecular techniques (e.g., optogenetics, chemogenetics) that can be used to probe and dissect with great precision the neural circuitry underlying motivational and emotional behavior in laboratory animals. Unfortunately, technical and conceptual innovations in the behavioral end points themselves, as modeled in non-human species, have not advanced at similar pace. The literature is replete with examples of elegant new molecular neurocircuitry techniques paired with behavioral end points such as time spent struggling during tail suspension, entries into the center of an open field, or latency to eat in a novel environment. Many of these tests are mischaracterized as disease "models" or are said to reflect depression or anxiety in mice or rats, whereas they are better characterized as assays that were developed and optimized to rapidly and inexpensively identify standard psychiatric medications (e.g., antidepressants, anxiolytics). These types of behavioral assays pose at least three major challenges. Foremost, they typically rely upon anthropomorphic inferences of how situations should affect the emotional state of the animal-often a mousewhich creates a degree of subjectivity that can make studies difficult to replicate, even among researchers working within the same lab. A second challenge is that behavioral tests performed in rodents are only rarely analogous to those that can be performed in humans, limiting translational value. Third, they are often brief tests that occur once at an arbitrary point in time; thus they are best considered "snapshots" that require fortuitous timing. The fact that these limitations represent major obstacles to developing therapeutics seems beyond debate, considering the obvious difficulties in developing mechanistically new drugs and the massive divestment by industry in CNS drug development. These factors have even led to questions about the utility of animal models in psychiatry research [16].

New approaches are emerging that reflect attempts to overcome these limitations. Some are generating digital phenotypinglike data sets that are directly analogous to those that can be collected in humans using wearable tracking devices or smart phones; end points such as locomotor activity, body temperature, sleep/wakefulness states, and vocalizations can now all be measured across phylogeny. Those with the greatest promise share three important qualities: they are objective (enabling identical conclusions across independent observers), continuous (obtaining large data sets over long periods of time), and translational (reflecting end points that are similar if not identical in humans and the model species). One example is the development of large-scale behavior-based systems [17] that use continuous tracking to measure locomotion, trajectory complexity, and simple behavioral sequences, collecting large numbers of data points (on the scale of hundreds of thousands) for each subject over long periods of time (on the scale of hours). These systems use machine learning to generate specific behavioral signatures of different classes and subclasses of drugs such as those with known anxiolytic or anxiogenic effects in humans, or compare known drug signatures to the effects of genetic manipulations. Other versions incorporate RDoC-relevant behavioral domains including social behavior, cognitive behavior, and circadian rhythms, and can provide insight on the probability of transitioning from one behavioral event to another. Such systems may be able to uncover complex behavioral patterns that would be impossible for human observers to detect, but represent fundamental "syllables" of rodent behavior [17]. Under the right 
circumstances, it may also be possible to identify antecedents that reliably predict at meaningful timescales the future occurrence of patterns (e.g., impulsivity) that represent risk factors for harmful behaviors (e.g., relapse to drug-seeking, suicide) (Fig. 3).

A second example of an end point with high translational relevance is sleep (or sleep quality), which is altered across various psychiatric illnesses. In humans, mood and anxiety disorders are associated with increased sleep latency and sleep disruption. It is currently unknown if sleep dysregulation precedes (or is a risk factor for) the development of these conditions, but it is clear that disrputed sleep exacerbates their pre-existing symptoms. In rodents, immobilization stress, footshock, and social defeat stress all affect sleep architecture, with numerous similarities to the human conditions [18, 19]. In addition, inflammatory processes-which have been broadly implicated in psychiatric illness [20]—can affect sleep end points [21]. Inflammation can also cause persistent effects on vocalization patterns in mice that are detectable with specialized microphones tuned to ultrasonic frequencies [22]. The fact that numerous types of stress produce alterations in sleep that are similar to those found in psychiatric illness suggests that examining sleep

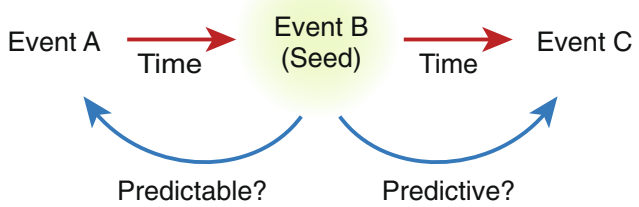

Fig. 3 Use of continuous data to predict behavior. In the example depicted, with behavior B serving as the reference ("Seed") Event, machine learning algorithms may be able to identify antecedents (i.e., Event $A$, which could be a behavior, an EEG signature, a temperature fluctuation, etc) that would have been predictive of Event $B$, and future behaviors (i.e., Event $C$ ) that are predictable on the basis of Event $B$ (or the combination of Events $A$ and $B$ ). Maximizing the timeframe between events within which predictions are reliable is critical and could be life-saving in neuropsychiatric conditions such as suicide or substance abuse architecture represents a currently underappreciated approach to identifying physiological biomarkers with translational relevance. Sleep can now be studied in great detail, under ethological (untethered) conditions, using wireless transmitters that are implanted subcutaneously and collect an uninterrupted stream of data for months, enabling longitudinal, within-subjects experimental designs that can provide detailed insight on the onset, recovery, and persistence of changes in sleep. These same systems also offer the ability to examine rhythms (diurnal, circadian) in locomotor activity and/or body temperature, as well as EEG spectral power, which may have potential as biomarkers for psychiatric conditions.

In humans, wearable devices and smart phones can collect data on locomotor activity body temperature, biological rhythms, sleep, speech patterns and cadences, and response control. As described above (Fig. 2), these end points represent key elements of the MSE: activity, speech, cognition. If homologous end points can be collected in rodents-activity, vocalizations, EEG spectra-and can be shown to be reliably sensitive to manipulations (e.g., stress) that cause or exacerbate psychiatric illness in humans, their use represents an innovative opportunity to better align basic and clinical research. Although insight on sleep in humans is often unavailable except via self-report, the easy availability of objective sleep metrics via devices may offer an added dimension to a modernized version of the digital MSE, and homologous data can be derived in laboratory animals via data sets that include EEG and EMG (electromyography). While more validation in laboratory animals is needed, use of a common set of end points (i.e., a crossspecies MSE: Fig. 4) and endophenotypes can more fully exploit the myriad advantages of basic research, including the ability to perform carefully controlled manipulations, study mechanism in exquisite detail, and establish cause-effect relationships. Effective use of common end points would represent the basis for screening methods that better predict effects that will be seen humans.

\section{CAVEATS}

While there is tremendous potential in this digital landscape to improve our science and healing abilities, there are also

\section{Digital MSE for rodents}
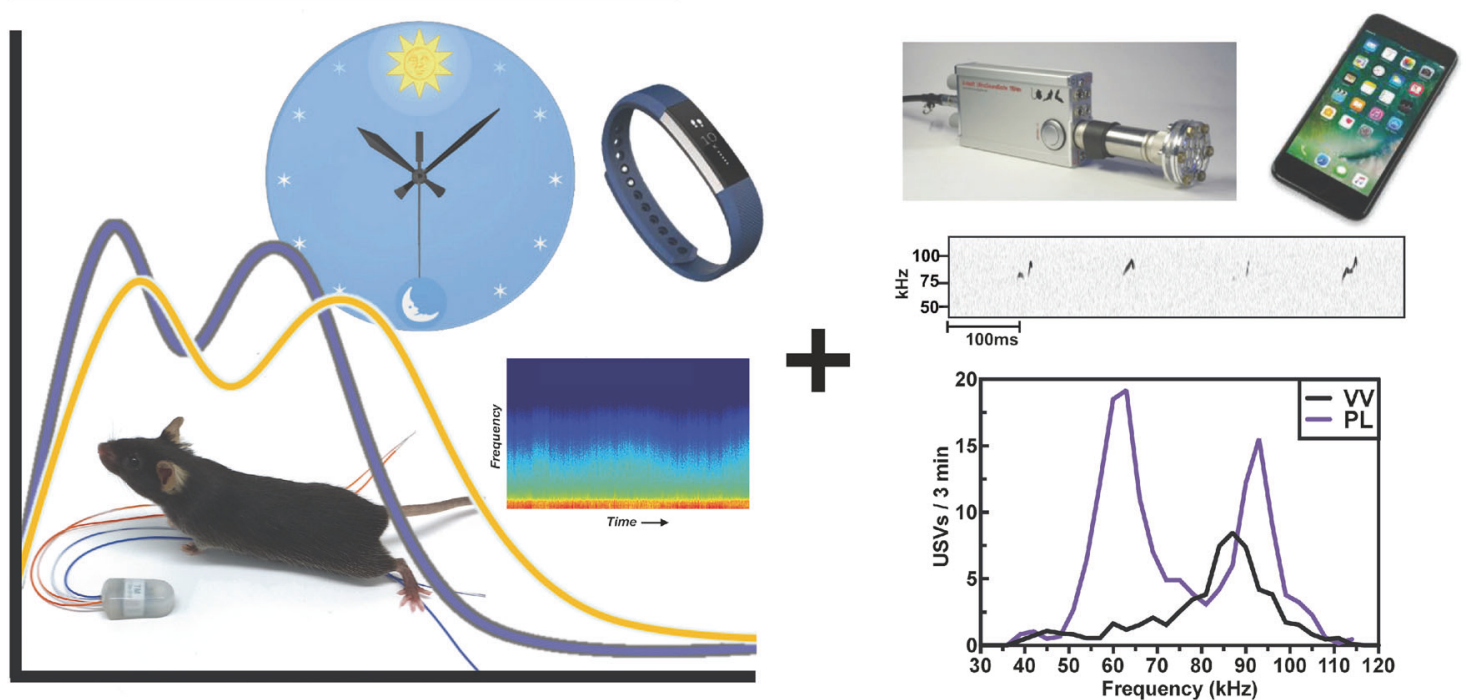

Fig. 4 Digital MSE for rodents, using end points that are objective, continuous, and translational. Research in laboratory animals is increasingly employing temporally dense behavioral recordings alongside neural data in ways that could translate to the human MSE. Sleep, diurnal and circadian rhythms, locomotor activity, and vocalizations can routinely be studied as a function of targeted interventions to expose new relationships among genes, circuits, systems, and complex behavior. Vocalizations courtesy of Galen Missig; VV: vehicle control; PL: perinatal immune-activated 
tremendous challenges inherent to working with deeply personal data of large numbers of individuals at scale and with the assistance of algorithms. Obviously, digital phenotyping as a medical approach requires permission from patients and/or family members, who are often eager to participate in programs that offer the possibility of relief from the debilitating symptoms of these currently intractable conditions. Carefully considering the ethical implications of any increased prevention capacity is critical for successful deployment; however, not investing in the development of such approaches because of a potential ethical issue would also be problematic, given the profound need for available services such systems could fulfill. Other ethical issues related to participant consent in longitudinal studies also require consideration as researchers begin assembling data sets and testing approaches. Finally, data quality concerns, such as how best to account for heterogeneity of hardware and software between individuals, or even within the same individual over time, are important challenges. These challenges can be met with established computational and experimental approaches: for instance, emphasizing stable features derived from unstable sensors (e.g., image stabilization on a camera), or capturing changes over time within an individual as opposed to relying on cross-sectional measures, although empirical data is required to test whether such approaches are viable under real-world conditions. We remain optimistic by anchoring our vision to the core root concept: that technology can and should be nothing more or less than the application of careful, systematic measurement-based approaches to the already well-established craft of working with individuals during times of mental suffering.

\section{SUMMARY}

The prevalence of wearable devices and smart phones, together with increasingly sophisticated computational approaches such as machine learning, can make extraordinarily large data sets available to psychiatrists, caregivers, and researchers. Beyond merely documenting compliance and representing methods of communicating with patients, they hold the promise of being able to identify antecedents with meaningful advance noticeminutes, hours, days-that allow patients to modify their behavior, or caregivers to intervene. Although important ethical and validation concerns remain, the ability to predict future behaviors on the basis of objective data would be transformative for conditions such as addiction, depression, and psychosis, where support systems are often effective in preventing catastrophic outcomes such as overdose and suicide. Areas of active research include understanding the ways in which behavioral events can predict one another, and the corresponding timescales for which the predictions are meaningful. Alignment of end points in humans and laboratory animals should be prioritized so that translationally relevant working hypotheses can be developed, tested, and validated, thereby ushering in a new era of more effective diagnoses, treatment, and prevention of psychiatric illness while eliminating the types of anthropomorphic interpretation of rudimentary behavior that promote broad skepticism regarding the utility of animal models. The application of new technologies to psychiatry and neuroscience has already enabled appreciation of cross-species homologies in neuroanatomy, neurophysiology, and neurogenetics. We are entering an era in which establishing the corresponding existence of behavioral homologies could have transformative impact on our ability to diagnose, treat, and prevent psychiatric illness.

\section{ACKNOWLEDGEMENTS}

We gratefully acknowledge the contributions of Nicholas Mirin, Sarah Vogel, Delores Nwokeafor-Laz and Niels Wright for assistance with figure preparation.

\section{ADDITIONAL INFORMATION}

Competing interests: JTB serves as a consultant for Pear Therapeutics and Niraxx Therapeutics, LTG serves as a consultant for 23andme, and KJR is on the Scientific Advisory Board for Resilience Therapeutics and serves as a consultant for Biogen. The other authors declare no competing interests.

Publisher's note: Springer Nature remains neutral with regard to jurisdictional claims in published maps and institutional affiliations.

\section{REFERENCES}

1. Naslund JA, Aschbrenner KA, Araya R, Marsch LA, Unützer J, Patel V, et al. Digital technology for treating and preventing mental disorders in low-income and middle-income countries: a narrative review of the literature. Lancet Psychiatry. 2017;4:486-500

2. Andrews G, Cuijpers P, Craske MG, McEvoy P, Titov N. Computer therapy for the anxiety and depressive disorders is effective, acceptable and practical health care: a meta-analysis. PloS ONE. 2010;5:e13196.

3. Yang N, Hing E. Table of Electronic Health Record adoption and use among office-based physicians in the U.S., by Specialty: 2015 National Electronic Health Records Survey. 2017. https://www.cdc.gov/nchs/data/ahcd/nehrs/ 2015_nehrs_ehr_by_specialty.pdf.

4. McCoy TH, Castro VM, Roberson AM, Snapper LA, Perlis RH. Improving prediction of suicide and accidental death after discharge from general hospitals with natural language processing. JAMA Psychiatry. 2016;73:1064-71.

5. Simon GE, Johnson E, Lawrence JM, Rossom RC, Ahmedani B, Lynch FL, et al. Predicting suicide attempts and suicide deaths following outpatient visits using electronic health records. Am J Psychiatry. 2018.

6. Busch $A B$, Bates DW, Rauch SL. Improving adoption of EHRs in psychiatric care. New Engl J Med. 2018;378:1665.

7. Onnela JP, Rauch SL. Harnessing smartphone-based digital phenotyping to enhance behavioral and mental health. Neuropsychopharmacology. 2016;41:1691.

8. Harati S, Crowell A, Mayberg H, Kong J, Nemati S. Discriminating clinical phases of recovery from major depressive disorder using the dynamics of facial expression. Conf Proc IEEE Eng Med Biol Soc. 2016:2254-57.

9. Rizzo A, Lucas G, Gratch J, Stratou G, Morency LP, Chavez K, et al. Automatic behavior analysis during a clinical interview with a virtual human. Stud Health Technol Inf. 2016;220:316.

10. Baker JT, Pennant L, Baltrušaitis T, Vijay S, Liebson ES, Ongur D, et al. Toward expert systems in mental health assessment: a computational approach to the face and voice in dyadic patient-doctor interactions. Iproceedings. 2016;2:e44.

11. Campbell K, Carpenter KL, Hashemi J, Espinosa S, Marsan S, Borg JS, et al. Computer vision analysis captures atypical attention in toddlers with autism. Autism. 2018.

12. Bedi G, Carrillo F, Cecchi GA, Slezak DF, Sigman M, Mota NB, et al. Automated analysis of free speech predicts psychosis onset in high-risk youths. npj Schizophr. 2015;1:15030.

13. Corcoran CM, Carrillo F, Fernández-Slezak D, Bedi G, Klim C, Javitt DC, et al. Prediction of psychosis across protocols and risk cohorts using automated language analysis. World Psychiatry. 2018;17:67.

14. Venek V, Scherer S, Morenc LP, Pestian J. Adolescent suicidal risk assessment in clinician-patient interaction. IEEE Trans Affect Comput. 2017;8:204.

15. Barnett I, Torous J, Staples P, Sandoval L, Keshavan M, Onnela JP. Relapse prediction in schizophrenia through digital phenotyping: a pilot study. Neuropsychopharmacology. 2018;43:1660.

16. Hyman S. Mental health: depression needs large human-genetics studies. Nature. 2014;515:189-91.

17. Markowitz JE, Gillis W, Beron C, Neufeld SQ, Roberston K, Bhaghat N, et al. The striatum organizes 3D behavior via moment-to-moment action selection. Cell 2018;174:44.

18. Lezak KR, Missig G, Carlezon WA Jr. Behavioral methods to study anxiety in rodents. Dialog- Clin Neurosci. 2017;19:79.

19. Wells AM, Ridener E, Bourbonais CA, Kim W, Pantazopoulos $\mathrm{H}$, Carroll Fl, et al Chronic socil stress-induced disruptions in sleep and circadian rhythms are mitigated by kappa-opioid receptor antagonism. J Neurosci. 2017;37:7656.

20. Miller $\mathrm{AH}$, Haroon $\mathrm{E}$, Felger JC. The immunology of behavior-exploring the role of the immune system in brain health and illness. Neuropsychopharmacology. 2017;42:1.

21. Missig G, Mokler EL, Robbins JO, Alexander AJ, McDougle CJ, Carlezon WA Jr. Perinatal immune activation produces persistent sleep alterations and epileptiform activity in male mice. Neurospychopharmacology. 2018;43:482-91.

22. Missig G, Robbins JO, Mokler EL, McDougle CJ, Carlezon WA Jr. Maternal immune activation with a TLR7 agonist results in sex-specific behavioral alterations in mice: relevance to neuropsychiatric disorders. Neuropsychopharmacology. 2017; 43:T108. 